\title{
White matter hyperintensities in migraine: a review
}

\author{
Mi Ji Lee ${ }^{1}$, Sujin Moon ${ }^{2}$, Chin-Sang Chung ${ }^{1}$ \\ ${ }^{1}$ Department of Neurology, Samsung Medical Center, Sungkyunkwan University School of Medicine, Seoul, Korea \\ ${ }^{2} \mathrm{CHA}$ University School of Medicine, Seongnam, Korea
}

Received: October 6, 2019

Revised: November 28, 2019

Accepted: December 3, 2019

Corresponding author:

Mi Ji Lee

Department of Neurology,

Samsung Medical Center,

Sungkyunkwan University

School of Medicine, 81 Irwon-

ro, Gangnam-gu, Seoul 06351, Korea

Tel: +82-2-3410-3599

E-mail:mijilee.md@gmail.com

This is an Open Access article distributed under the terms of the Creative Commons Attribution Non-Commercial License (http:// creativecommons.org/licenses/ by-nc/4.0/).

\begin{abstract}
Migraine is a neurological disorder characterized by recurrent spells of headache accompanied by brain symptoms such as nausea, vomiting, photophobia, and phonophobia. White matter hyperintensity (WMH) is the most frequent radiological abnormality observed in migraineurs. Older studies have reported that the prevalence of WMH is about 30\% in migraineurs, while latest studies using advanced technology show that WMHs are present in about $70 \%$ of young (age $<50$ years), vascular risk factor-free patients with migraine. Population-based studies on elderlies reported conflicting results on the association between migraine and WMH, but deep WMHs were greater in studies of the younger population. Data are also conflicting on whether headache characteristics, migraine type, disease duration, or headache frequency are associated with WMH. Current evidence suggests that a greater deep WMH burden is associated with intracranial and extracranial vascular function. The clinical implication of WMH has not been well studied in relation to migraine, but it is generally not associated with cognitive dysfunction in patients with migraine. Current understanding of WMHs is limited; we herein suggest some tips for improvement in research methods that will bring a better understanding of both the pathophysiology and the consequence of WMH development in patients with migraine.
\end{abstract}

Keywords: Ischemia; Magnetic resonance imaging; Migraine with aura; Migraine without aura; Stroke; White matter

\section{INTRODUCTION}

Migraine is a neurological disorder characterized by recurrent episodes of headache that is accompanied by brain symptoms such as nausea, vomiting, photophobia, and phonophobia. About $10 \%$ of the world's population has migraine [1]. The impact of migraine is substantial; it has been ranked among the top leading causes of disability in the Global Burden of Disease study 2016 [2].

Not only is migraine a disorder causing headache-related impact, it also contributes to a higher risk of cardiovascular outcomes such as stroke, ischemic heart disease, and all-cause death [3-7]. The association between migraine and stroke has been particularly well recog- 
nized. Several population-based studies reported an increased risk of stroke in migraineurs. Especially, subgroups of migraine with aura (MWA) and female migraineurs carry a higher risk of ischemic and hemorrhagic strokes $[4,6]$.

However, clinical or translational studies on the mechanism and prevention of stroke in migraineurs have rarely been conducted. This is because the absolute incidence of stroke is low; hence, a long observation period and a huge sample size are required in observational studies using stroke as an outcome. Instead, understanding the white matter hyperintensity (WMH) may contribute to knowledge about the mechanism of ischemic stroke in migraineurs. WMH refers to abnormal signal changes in white matter in T2-based brain magnetic resonance imaging (MRI) [8]. The pathophysiology of WMH is not exactly understood and may be heterogenous, but ischemic insult is the most likely mechanism [9].

In this paper, we will review the association between WMH and migraine, possible mechanisms, and the current limitations of knowledge in this field.

\section{WHAT IS WMH?}

WMH is a radiological term indicating the areas with increased signal intensity on T2-weighted MR imaging $[8,10]$. Although WMH is currently defined based on MRI findings, white matter changes have been recognized since the late 1980s with the use of brain computed tomography (CT) [11]. Low-attenuated white matter areas on CT were called leukoaraiosis [12]. With advances in neuroimaging techniques, the identification of WMH is easier and more sensitive than before.

As mentioned above, WMH is hyperintense on T2-weighted images and best detected on fluid-attenuated inversion recovery (FLAIR) sequence. Typical WMH is round- or oval-shaped, hypo or isointense on T1-weighted images and is distributed in the periventricular or deep white matter [8,9]. WMH should be differentiated from enlarged perivascular space (Virchow-Robin space), lacunes [13], and other disorders such as multiple sclerosis that can cause white matter lesions [9].

\section{EPIDEMIOLOGY}

WMH is prevalent in the general population and has thus been a frequent cause of neurology consultation in people who undergo brain MRI. The incidence of WMH increases with age, especially after midlife. The reported prevalence of WMH differs between studies because the detection of WMH is highly dependent on MRI resolution and the size criteria. In modern studies, the prevalence of WMH is about $50 \%$ in the general population aged 44 to 48 years and increases to $90 \%$ in people aged 64 to 74 years [14]. In contrast, younger people do not frequently exhibit WMHs. The risk of having WMH is tenfold higher in people aged 55 years or older compared to that in those aged less than that [15].

\section{TYPES OF WMH: PERIVENTRICULAR WMH AND DEEP WMH}

WMHs are classified into two types: periventricular and deep WMHs. Periventricular WMHs refer to hyperintensities surrounding the ventricles, and deep WMHs are located in deep and juxtacortical white matter $[10,16,17]$. Risk factors and clinical implications may be different between the two types [18]. While WMH has been recognized in demented patients, and age and hypertension are well known risk factors, periventricular WMH is more strongly associated with age, hypertension, and cognitive decline than deep WMH [18-21]. In contrast, migraine is more associated with deep WMH $[18,22,23]$. Different pathologies are also observed; although controversial data exists, most studies indicate that ischemic and hypoxic pathologies are predominant in deep WMHs, while periventricular WMHs are more linked to increased interstitial fluid probably caused by altered periventricular fluid dynamics [24,25]. In this review article, the association between migraine and WMH will be discussed with regard to the type of WMHs.

\section{MIGRAINE AND WMH}

While conventional neuroimaging of migraine reveals no diagnostic findings, WMHs may be the most frequent abnormalities observed in migraineurs. Patients often ask about the clinical implication of these lesions and their association with migraine. However, as WMHs are also common among the general population of middle-aged and elderlies, it is not easy to determine whether the increased prevalence of WMHs in migraineurs is attributed to either migraine or the normal aging process. Even if WMHs are purely migraine-related, they may be obscured by age-related changes in susceptible age groups. In addition, migraine patients with WMHs are more likely to visit secondary or tertiary hospitals to seek a second opinion, which may lead to overestimation of its prevalence in migraineurs. Therefore, the study on WMH in migraineurs should be interpreted with regard to age and study setting. 
PRECISION AND FUTURE MEDICINE

White matter hyperintensities in migraine

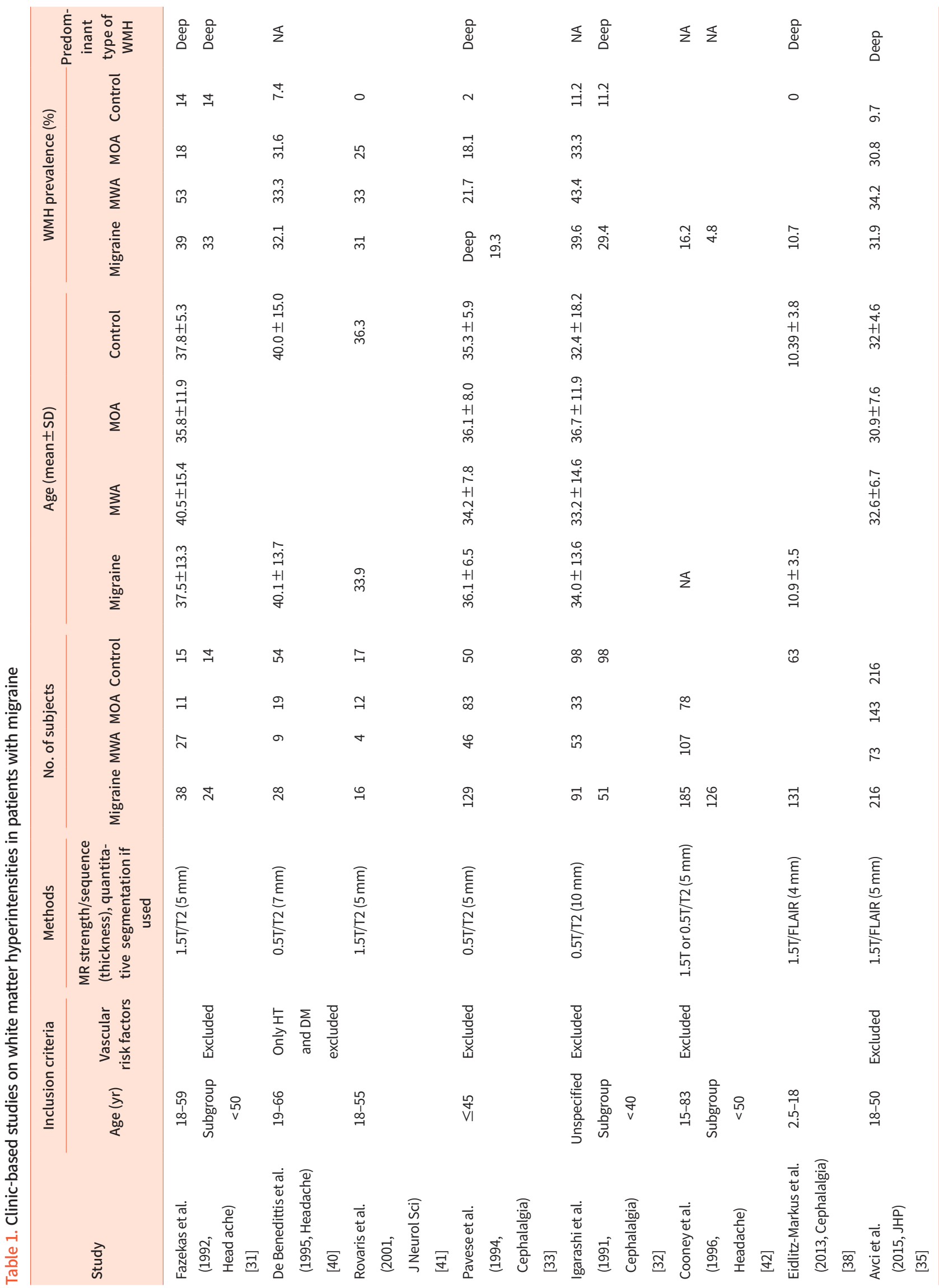




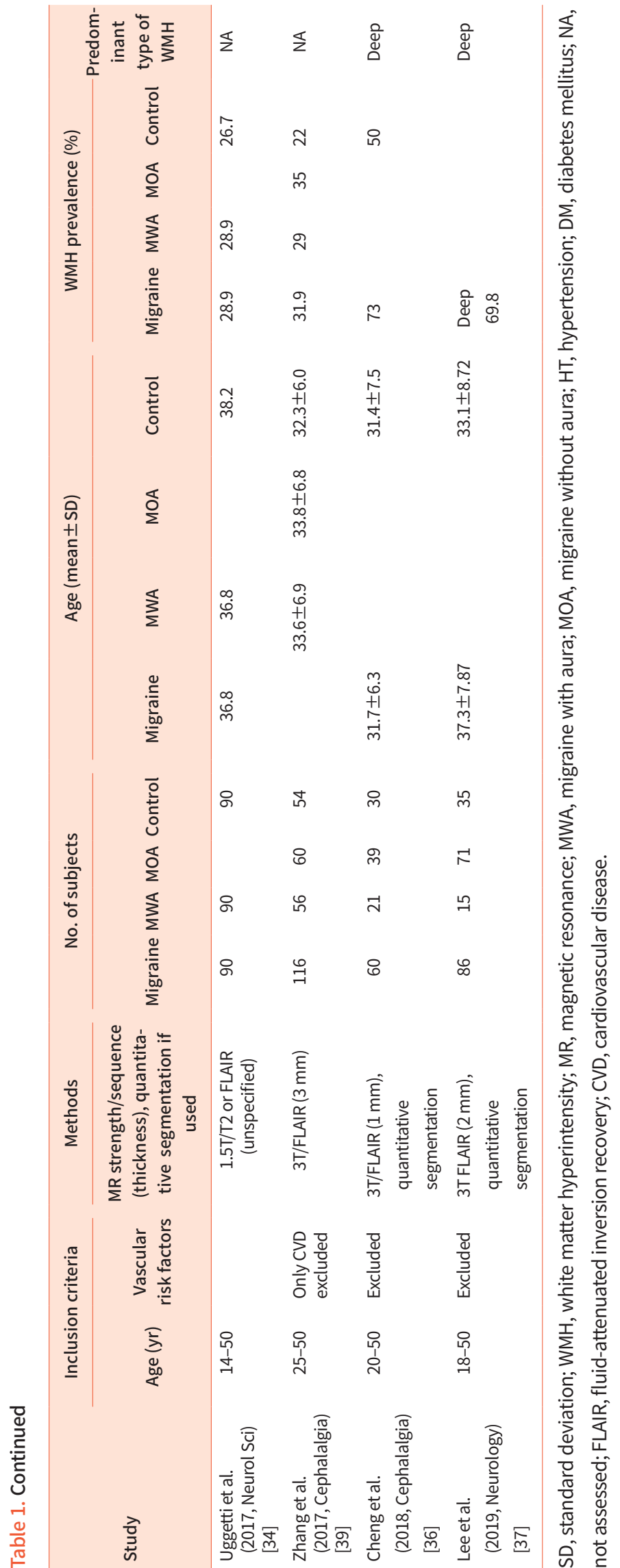

Cerebral autosomal dominant arteriopathy with subcortical infarcts and leukoencephalopathy (CADASIL) is an autosomal dominant disorder causing recurrent strokes and extensive WMHs [26]. CADASIL is an important differential diagnosis when patients have severe headaches and WMHs, since about $30 \%$ to $40 \%$ of CADASIL patients can manifest migraine headaches [26,27]. In autopsy specimens of CADASIL, myelin loss and atrophy of the white matter can be found [28,29]. Vascular ultrastructural changes such as the loss of medial smooth muscle cells, adventitial fibrosis, and consequent vascular hypotonicity are prominent findings in CADASIL $[28,29]$. WMHs in CADASIL appear earlier in life and more extensively than in migraine. Significant white matter lesion load before the age of 35 , multiple lacunar infarcts, and characteristic involvement of anterior temporal pole and external capsule suggest the possibility of CADASIL in patients with migraine [30].

\section{Increased WMH in patients with migraine: clinic- based studies}

Several clinic-based studies have reported that migraineurs have an increased burden of WMHs (Table 1) [31-42]. When restricted to patients aged $<50$ years and without vascular risk factors, earlier studies using 0.5 or 1.5 Tesla MRI consistently reported about $30 \%$ prevalence of $\mathrm{WMH}$ in patients with migraine [31-35]. Meta-analysis of earlier studies showed a 3.9-fold increase in the odds of WMHs being present in patients with migraine as compared to controls [43]. Recent studies using higher resolution MRI (3T, slice thickness of $<3 \mathrm{~mm}$ ) and quantitative segmentation methods showed a higher prevalence of WMH than was reported earlier; it is about $70 \%$ in vascular risk factor-free migraine patients aged $<50$ years $[36,37]$. WMHs are also present in childhood migraine. In a study from a pediatric clinic, WMHs were found in $10 \%$ of pediatric patients with migraine, but not in patients with other neurological disorders [38].

\section{Increased WMH in patients with migraine: popula- tion-based studies}

There are six population-based studies on the risk of WMH in migraineurs (Table 2) [22,23,44-47]. In these studies, the study population was older than those included in the clinic-based studies that were mostly middle-aged or elderlies. Among these, study participants in the Cerebral Abnormalities in Migraine: An Epidemiological Risk Analysis Study 1 (CAMERA-1) were relatively younger (mean age, 48 years) than those who were included in the other studies [22]. The types of WMH were specified as periventricular and deep in 


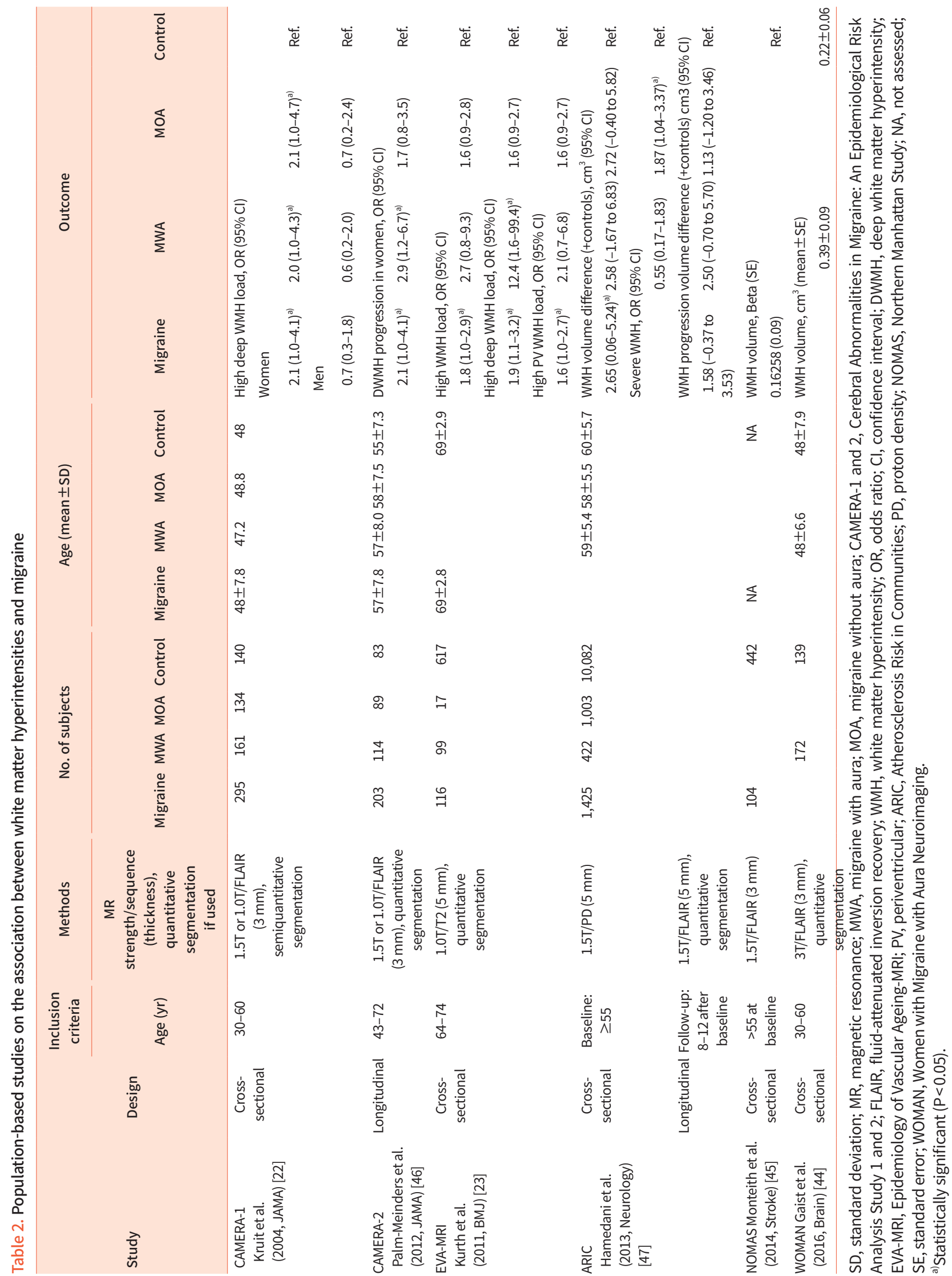


CAMERA-1, 2, Epidemiology of Vascular Ageing-MRI (EVA$M R I)$, and Women with Migraine with Aura Neuroimaging (WOMAN) studies, whereas Atherosclerosis Risk in Communities (ARIC) study, and Northern Manhattan Study (NOMAS) only measured total WMHs $[22,23,44-47]$.

Among cross-sectional studies, three reported an increased burden of WMH in migraineurs. CAMERA-1 study reported a two-fold increase in the risk of high deep WMH load in female migraineurs [22]. EVA-MRI study showed an increased risk of all WMHs (odds ratio [OR], 1.8), deep WMHs $(\mathrm{OR}, 1.9)$, and periventricular WMHs $(\mathrm{OR}, 1.6)$ in migraineurs [23]. ARIC study did not report OR or hazard ratio, but the WMH volume was significantly greater in migraineurs than in controls [47]. In contrast, NOMAS and WOMAN studies reported no association between migraine and WMHs [44,45].

Among two longitudinal studies (CAMERA-2 and ARIC follow-up studies), CAMERA-2 reported a more rapid progression of deep WMH in female migraineurs than in controls [46]. The ARIC follow-up study also showed greater changes in WMH in migraineurs, but the association became insignificant when adjusted for demographics and vascular risk factors [47].

\section{Risk of WMH in patients with migraine: summary}

Most of the clinic-based studies show an increased risk of WMH in migraineurs, and deep WMH was the predominant type. Possible caveats of clinic-based studies are selection bias and referral bias; the findings might be from those who were severely affected. In contrast, population-based studies show conflicting results on the risk of WMH in migraine. However, studies that recruited a relatively young population and focused on deep WMHs were more likely to have positive results. Taken together, these studies may suggest that migraineurs develop WMHs earlier than migraine-free controls, particularly in the deep white matter, but the differences may be overwhelmed by age-related changes after middle age. Whether the increased burden of WMHs is caused by migraine or its comorbidities is still unclear, but the CAMERA-2 study suggests a possible effect of migraine itself on the risk of deep WMH at least in women.

\section{FACTORS ASSOCIATED WITH WMH IN MIGRAINEURS}

Results from clinic-based studies provide valuable information about the detailed features, clinical correlation, and neurophysiologic correlates of WMH. In studies specifying the type of WMHs, deep WMHs were more prevalent in pa- tients with migraine than in migraine-free controls, while periventricular WMHs did not differ much between patients and controls $[32,33,35-37,48]$. Studies regarding clinical correlates of WMH did not yield consistent findings; disease duration and attack frequency were significantly associated with WMH or decreased WM integrity in some studies [34, $36,49,50]$, while others did not show such an association [37,39]. Although the earlier study of Fazekas et al. [31] reported a higher prevalence of WMH in patients with MWA compared to that in those with migraine without aura, modern studies have shown no such difference [34,35,39,50]. One study reported that the dominant side of WMH matched to the dominant side of headache [51].

Studies on the pathophysiology of WMH in migraine are scarce. Currently, two studies recruited young (age $<50$ years) patients with migraine without vacular risk factors and normal controls to investigate the relationship between vascular function and WMH. One study showed reduced cerebrovascular reactivity was associated with high deep WMH burden only in patients with migraine [37]. In this study, cerebrovascular reactivity was significant determinant of the deep WMH number, while age was associated with deep WMH volume [37]. The other study reported systemic arterial stiffness and lower carotid pulsatility index were associated with WMH load in patients with migraine [36]. In this study, systolic blood pressure was also significant determinant of WMH volume. Given that migraine is a disorder of cerebral hyperexcitability, these findings suggest cerebral and systemic vascular dysfunction may cause relative cerebral ischemia when the cerebral metabolic demands become increased and exaggerated vasodilatory response are required. This theory can be one of the disease-specific mechanism of WMH in migraineurs [36,37]. In addition, aging and hypertension may cause WMHs in migraineurs as they do in general population.

Right-to-left shunt (RLS) has been suggested as a possible mechanism of stroke, silent infarct, and WMH in migraine patients. However, study results were highly controversial; while several studies did not show an association between the presence of RLS and WMH [52-55], some studies reported an association between RLS and certain types of WMHs in migraineurs [56-58].

\section{CLINICAL IMPLICATION}

A meta-analysis showed that WMHs are associated with an increased risk of cognitive decline, incident dementia, ischemic stroke, and death [59]. However, there is no study on 
the effect modification of WMH on migraine-specific risk of neurologic outcomes. Migraineurs are at an increased risk of ischemic stroke, but the effect of WMH on subsequent stroke has not been investigated yet. Longitudinal studies reported no increased risk of incident dementia in migraineurs, and even a better cognition was noted in migraineurs [60-62].

Studies on WMHs and migraine outcome are also scarce. One study investigated the clinical course of migraine according to the presence of WMH [63]. In this study, baseline WMHs were more frequent in patients who did not show an improvement in migraine frequency after 3 years. Further studies are needed to draw a conclusion on the clinical impact of WMH in patients with migraine.

\section{CURRENT CHALLENGES OF WMH RE- SEARCH IN MIGRAINE}

\section{Methodological issue: MRI acquisition}

Previous studies used lower intensity (1.0 or 1.5T) MR machines and $\mathrm{T} 2$ or proton density sequences. However, such an acquisition might be less sensitive to detect small deep WMHs and less specific to WMHs; lacunes and perivascular spaces might not be clearly distinguished by using these protocols. For research on WMHs, minimal requisition is the FLAIR sequence with a slice thickness of 3 to $5 \mathrm{~mm}$ and an inplane resolution of $1 \mathrm{~mm}$. T1- and T2-weighted images are further required for discriminating lacunes and perivascular spaces from WMHs [8]. Recently, an isotropic volumetric 3D FLAIR with at least 1-mm isotropic voxels is considered to have the best sensitivity and specificity for advanced research [8].

\section{Methodological issue: detection of WMH}

Initial studies of WMH used manual counting and grading, which might be suitable for studies with small sample sizes. However, this method is less applicable for larger studies as it has an inter-rater/intra-rater reliability issue and lacks quantitative measurements. Therefore, several quantitative segmentation methods have been developed in the field of dementia research. Most of them are semi-automated and require a manual correction because erroneous results such as artifacts, lacunes, perivascular spaces, or white matter lesions from other disorders cannot be reliably excluded when automated detection is used. As the sensitivity of WMH detection increases, the rate of such false-positive lesions also increases. Currently available detection methods are less optimal for detection of small, juxtacortical, or low-intensity
WMHs that are predominantly seen in migraineurs.

We compared several preexisting segmentation methods for detecting small deep WMHs in migraineurs aged 18 to 50 years, and their performance were poor in sensitivity and specificity [64]. Thus, we have developed a fully automated, machine learning-based, segmentation method for detecting small deep WMH in migraineurs and showed its superiority compared to pre-existing segmentation tools (Fig. 1) [64]. Recently, we also developed a deep learning-based detection method for the detection of small deep WMHs in migraineurs and obtained a slightly better result [65]. We hope these methodological advances will improve the research of $\mathrm{WMH}$ in the field of migraine.

Diffusion tensor imaging and magnetization transfer imaging (MTI) have been widely used to evaluate the white matter integrity in disorders affecting white matter [66-68]. In the field of migraine, a recent analysis of CAMERA study using MTI showed that microstructural alteration of normal-appearing white matter in migraineurs before WMHs become apparently visible in the corresponding region [69]. These techniques can enhance the detection of white matter abnormality in short-term scale studies.

\section{Limitations in the research on WMH pathophysiology in migraine}

As discussed earlier, a number of studies investigated the epidemiological association between migraine and WMH. Nevertheless, studies on WMH pathophysiology and development are relatively scarce in the field of migraine. This might be attributable to the fact that younger migraineurs should be investigated for the research on WMH development, but the prevalence or development rate of WMHs might be low in such a study population to enable longitudinal observation. Furthermore, longitudinal data on normal controls are lacking in younger people. A longitudinal study on WMH development in younger migraineurs (mean age, 34.5 years; range, 19 to 49) and age- and sex-matched headache-free controls is ongoing at our center (ClinicalTrials.gov Identifier: NCT03494673). We hope that this study will provide important information about the prevalence, progression rate, and pathophysiology of WMHs in young migraineurs.

The dynamic contrast-enhanced MRI (DCE-MRI) technique can enable the evaluation of blood-brain barrier integrity [70]. Although the pre-existing evidence of blood-brain barrier leakage is poor in terms of ictal changes [71,72], an interictal DCE-MRI study suggested a regional alteration of bloodbrain barrier permeability [73]. This may be applicable in re- 


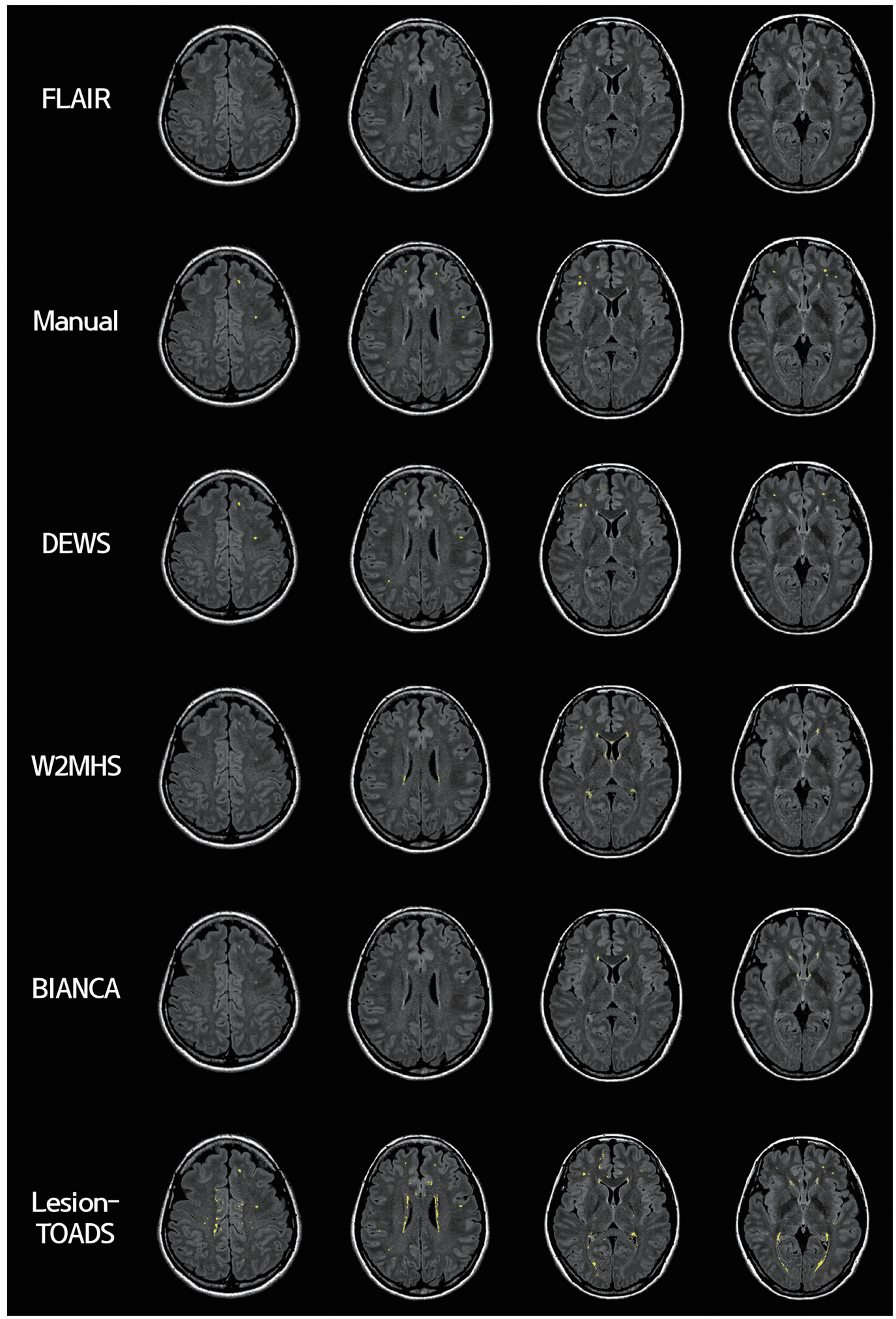

Fig. 1. Comparison of results from several segmentation methods. 1st row, the fluid-attenuated inversion recovery (FLAIR) image corrected for magnetic field bias; 2nd row, manually drawn deep white matter hyperintensity (WMH) clusters (in yellow); 3rd row, deep WMH clusters segmented using our new pipeline (DEep White matter hyperintensity Segmentation framework [DEWS]), 4th to 6th rows, deep WMH clusters segmented using pre-existing segmentation pipelines: Wisconsin WMH Segmentation Toolbox (W2MHS), Brain Intensity AbNormality Classification Algorithm (BIANCA), and Lesion-TOpology-preserving Anatomical Segmentation (Lesion-TOADS), respectively. Adapted from Park et al. [64]. 
search on the WMH pathophysiology. However, a reliability issue on the application of DCE-MRI to the white matter should be addressed.

\section{CONCLUSION}

WMHs are common radiological abnormalities that are encountered in headache clinics. In this paper, the association between migraine and WMH as well as their risk factors have been reviewed. It is important that physicians be made aware of the possible pitfalls and controversies related to the association between migraine and $\mathrm{WMH}$. WMH is a valuable marker for investigating brain ischemia in migraineurs; thus, further studies on the pathophysiology of WMH are needed.

\section{CONFLICTS OF INTEREST}

No potential conflict of interest relevant to this article was reported.

\section{ACKNOWLEDGMENTS}

This work was supported by the National Research Foundation of Korea (NRF) grant funded by the Korea government (MSIP) (No. 2017R1A2B4007254 and 2017R1A2B2009086).

\section{ORCID}

Mi Ji Lee $\quad$ https://orcid.org/0000-0003-1364-1969

Sujin Moon https://orcid.org/0000-0002-2064-7923

Chin-Sang Chung https://orcid.org/0000-0002-0049-558X

\section{REFERENCES}

1. Woldeamanuel YW, Cowan RP. Migraine affects 1 in 10 people worldwide featuring recent rise: a systematic review and meta-analysis of community-based studies involving 6 million participants. J Neurol Sci 2017;372:30715.

2. GBD 2016 Disease and Injury Incidence and Prevalence Collaborators. Global, regional, and national incidence, prevalence, and years lived with disability for 328 diseases and injuries for 195 countries, 1990-2016: a systematic analysis for the Global Burden of Disease Study 2016. Lancet 2017;390:1211-59.

3. Hu X, Zhou Y, Zhao H, Peng C. Migraine and the risk of stroke: an updated meta-analysis of prospective cohort studies. Neurol Sci 2017;38:33-40.

4. Sacco S, Ornello R, Ripa P, Pistoia F, Carolei A. Migraine and hemorrhagic stroke: a meta-analysis. Stroke 2013; 44:3032-8.

5. Schurks M, Rist PM, Bigal ME, Buring JE, Lipton RB, Kurth T. Migraine and cardiovascular disease: systematic review and meta-analysis. BMJ 2009;339:b3914.

6. Spector JT, Kahn SR, Jones MR, Jayakumar M, Dalal D, Nazarian S. Migraine headache and ischemic stroke risk: an updated meta-analysis. Am J Med 2010;123:612-24.

7. Sacco S, Ornello R, Ripa P, Tiseo C, Degan D, Pistoia F, et al. Migraine and risk of ischaemic heart disease: a systematic review and meta-analysis of observational studies. Eur J Neurol 2015;22:1001-11.

8. Wardlaw JM, Smith EE, Biessels GJ, Cordonnier C, Fazekas F, Frayne R, et al. Neuroimaging standards for research into small vessel disease and its contribution to ageing and neurodegeneration. Lancet Neurol 2013;12: 822-38.

9. Wardlaw JM, Smith C, Dichgans M. Mechanisms of sporadic cerebral small vessel disease: insights from neuroimaging. Lancet Neurol 2013;12:483-97.

10. Fazekas F, Chawluk JB, Alavi A, Hurtig HI, Zimmerman RA. MR signal abnormalities at $1.5 \mathrm{~T}$ in Alzheimer's dementia and normal aging. AJR Am J Roentgenol 1987;149:351-6.

11. Wardlaw JM, Valdes Hernandez MC, Munoz-Maniega S. What are white matter hyperintensities made of? Relevance to vascular cognitive impairment. J Am Heart Assoc 2015;4:001140.

12. Hachinski VC, Potter P, Merskey H. Leuko-araiosis. Arch Neurol 1987;44:21-3.

13. Kwee RM, Kwee TC. Virchow-Robin spaces at MR imaging. Radiographics 2007;27:1071-86.

14. Soderlund H, Nyberg L, Adolfsson R, Nilsson LG, Launer LJ. High prevalence of white matter hyperintensities in normal aging: relation to blood pressure and cognition. Cortex 2003;39:1093-105.

15. Hopkins RO, Beck CJ, Burnett DL, Weaver LK, Victoroff J, Bigler ED. Prevalence of white matter hyperintensities in a young healthy population. J Neuroimaging 2006;16: 243-51.

16. Brant-Zawadzki M, Fein G, Van Dyke C, Kiernan R, Davenport L, de Groot J. MR imaging of the aging brain: patchy white-matter lesions and dementia. AJNR Am J Neuroradiol 1985;6:675-82.

17. Zimmerman RD, Fleming CA, Lee BC, Saint-Louis LA, Deck MD. Periventricular hyperintensity as seen by magnetic 
resonance: prevalence and significance. AJR Am J Roentgenol 1986;146:443-50.

18. Rostrup E, Gouw AA, Vrenken H, van Straaten EC, Ropele $S$, Pantoni L, et al. The spatial distribution of age-related white matter changes as a function of vascular risk factors: results from the LADIS study. Neuroimage 2012;60: 1597-607.

19. Griffanti L, Jenkinson M, Suri S, Zsoldos E, Mahmood A, Filippini $\mathrm{N}$, et al. Classification and characterization of periventricular and deep white matter hyperintensities on MRI: a study in older adults. Neuroimage 2018;170: 174-81.

20. van Dijk EJ, Prins ND, Vrooman HA, Hofman A, Koudstaal PJ, Breteler MM. Progression of cerebral small vessel disease in relation to risk factors and cognitive consequences: Rotterdam Scan study. Stroke 2008;39:2712-9.

21. Seo SW, Lee JM, Im K, Park JS, Kim SH, Kim ST, et al. Cortical thinning related to periventricular and deep white matter hyperintensities. Neurobiol Aging 2012;33:1156-67.

22. Kruit MC, van Buchem MA, Hofman PA, Bakkers JT, Terwindt GM, Ferrari MD, et al. Migraine as a risk factor for subclinical brain lesions. JAMA 2004;291:427-34.

23. Kurth T, Mohamed S, Maillard P, Zhu YC, Chabriat H, Mazoyer $\mathrm{B}$, et al. Headache, migraine, and structural brain lesions and function: population based Epidemiology of Vascular Ageing-MRI study. BMJ 2011;342:c7357.

24. Fazekas F, Kleinert R, Offenbacher H, Schmidt R, Kleinert G, Payer F, et al. Pathologic correlates of incidental MRI white matter signal hyperintensities. Neurology 1993;43: 1683-9.

25. Fernando MS, Simpson JE, Matthews F, Brayne C, Lewis CE, Barber R, et al. White matter lesions in an unselected cohort of the elderly: molecular pathology suggests origin from chronic hypoperfusion injury. Stroke 2006;37:13918.

26. Dichgans M, Mayer M, Uttner I, Bruning R, Muller-Hocker J, Rungger $\mathrm{G}$, et al. The phenotypic spectrum of CADASIL: clinical findings in 102 cases. Ann Neurol 1998;44:731-9.

27. Ceroni M, Poloni TE, Tonietti S, Fabozzi D, Uggetti C, Frediani F, et al. Migraine with aura and white matter abnormalities: Notch3 mutation. Neurology 2000;54:1869-71.

28. Okeda R, Arima K, Kawai M. Arterial changes in cerebral autosomal dominant arteriopathy with subcortical infarcts and leukoencephalopathy (CADASIL) in relation to pathogenesis of diffuse myelin loss of cerebral white matter: examination of cerebral medullary arteries by reconstruction of serial sections of an autopsy case. Stroke
2002;33:2565-9.

29. Brulin P, Godfraind C, Leteurtre E, Ruchoux MM. Morphometric analysis of ultrastructural vascular changes in CADASIL: analysis of 50 skin biopsy specimens and pathogenic implications. Acta Neuropathol 2002;104:241-8.

30. Gladstone JP, Dodick DW. Migraine and cerebral white matter lesions: when to suspect cerebral autosomal dominant arteriopathy with subcortical infarcts and leukoencephalopathy (CADASIL). Neurologist 2005;11:1929.

31. Fazekas F, Koch M, Schmidt R, Offenbacher H, Payer F, Freidl $\mathrm{W}$, et al. The prevalence of cerebral damage varies with migraine type: a MRI study. Headache 1992;32:28791.

32. Igarashi H, Sakai F, Kan S, Okada J, Tazaki Y. Magnetic resonance imaging of the brain in patients with migraine. Cephalalgia 1991;11:69-74.

33. Pavese N, Canapicchi R, Nuti A, Bibbiani F, Lucetti C, Collavoli $\mathrm{P}$, et al. White matter MRI hyperintensities in a hundred and twenty-nine consecutive migraine patients. Cephalalgia 1994;14:342-5.

34. Uggetti C, Squarza S, Longaretti F, Galli A, Di Fiore P, Reganati PF, et al. Migraine with aura and white matter lesions: an MRI study. Neurol Sci 2017;38:11-3.

35. Avci AY, Lakadamyali H, Arikan S, Benli US, Kilinc M. High sensitivity C-reactive protein and cerebral white matter hyperintensities on magnetic resonance imaging in migraine patients. J Headache Pain 2015;16:9.

36. Cheng CY, Cheng HM, Chen SP, Chung CP, Lin YY, Hu HH, et al. White matter hyperintensities in migraine: clinical significance and central pulsatile hemodynamic correlates. Cephalalgia 2018;38:1225-36.

37. Lee MJ, Park BY, Cho S, Park H, Chung CS. Cerebrovascular reactivity as a determinant of deep white matter hyperintensities in migraine. Neurology 2019;92:e342-50.

38. Eidlitz-Markus T, Zeharia A, Haimi-Cohen Y, Konen O. MRI white matter lesions in pediatric migraine. Cephalalgia 2013;33:906-13.

39. Zhang Q, Datta R, Detre JA, Cucchiara B. White matter lesion burden in migraine with aura may be associated with reduced cerebral blood flow. Cephalalgia 2017;37: 517-24.

40. De Benedittis G, Lorenzetti A, Sina C, Bernasconi V. Magnetic resonance imaging in migraine and tension-type headache. Headache 1995;35:264-8.

41. Rovaris M, Bozzali M, Rocca MA, Colombo B, Filippi M. An MR study of tissue damage in the cervical cord of patients 
with migraine. J Neurol Sci 2001;183:43-6.

42. Cooney BS, Grossman RI, Farber RE, Goin JE, Galetta SL. Frequency of magnetic resonance imaging abnormalities in patients with migraine. Headache 1996;36:616-21.

43. Swartz RH, Kern RZ. Migraine is associated with magnetic resonance imaging white matter abnormalities: a meta-analysis. Arch Neurol 2004;61:1366-8.

44. Gaist D, Garde E, Blaabjerg M, Nielsen HH, Kroigard T, Ostergaard $\mathrm{K}$, et al. Migraine with aura and risk of silent brain infarcts and white matter hyperintensities: an MRI study. Brain 2016;139:2015-23.

45. Monteith T, Gardener H, Rundek T, Dong C, Yoshita M, Elkind MS, et al. Migraine, white matter hyperintensities, and subclinical brain infarction in a diverse community: the northern Manhattan study. Stroke 2014;45:1830-2.

46. Palm-Meinders IH, Koppen H, Terwindt GM, Launer LJ, Konishi J, Moonen JM, et al. Structural brain changes in migraine. JAMA 2012;308:1889-97.

47. Hamedani AG, Rose KM, Peterlin BL, Mosley TH, Coker LH, Jack CR, et al. Migraine and white matter hyperintensities: the ARIC MRI study. Neurology 2013;81:1308-13.

48. Seneviratne U, Chong W, Billimoria PH. Brain white matter hyperintensities in migraine: clinical and radiological correlates. Clin Neurol Neurosurg 2013;115:1040-3.

49. Schmitz N, Admiraal-Behloul F, Arkink EB, Kruit MC, Schoonman GG, Ferrari MD, et al. Attack frequency and disease duration as indicators for brain damage in migraine. Headache 2008;48:1044-55.

50. Trauninger A, Leel-Ossy E, Kamson DO, Poto L, Aradi M, Kover F, et al. Risk factors of migraine-related brain white matter hyperintensities: an investigation of 186 patients. J Headache Pain 2011;12:97-103.

51. Yalcin A, Ceylan M, Bayraktutan OF, Akkurt A. Episodic migraine and white matter hyperintensities: association of pain lateralization. Pain Med 2018;19:2051-7.

52. Adami A, Rossato G, Cerini R, Thijs VN, Pozzi-Mucelli R, Anzola GP, et al. Right-to-left shunt does not increase white matter lesion load in migraine with aura patients. Neurology 2008;71:101-7.

53. Del Sette M, Dinia L, Bonzano L, Roccatagliata L, Finocchi C, Parodi RC, et al. White matter lesions in migraine and right-to-left shunt: a conventional and diffusion MRI study. Cephalalgia 2008;28:376-82.

54. Jiang XH, Wang SB, Tian Q, Zhong C, Zhang GL, Li YJ, et al. Right-to-left shunt and subclinical ischemic brain lesions in Chinese migraineurs: a multicentre MRI study. BMC Neurol 2018;18:18.
55. Koppen H, Palm-Meinders IH, Mess WH, Keunen RW, Terwindt GM, Launer LJ, et al. Systemic right-to-left shunts, ischemic brain lesions, and persistent migraine activity. Neurology 2016;86:1668-75.

56. Yoon GJ, Kim JT, Chang J, Kim DE, Cho BH, Lee JH, et al. Right-to-left shunts as a cause of juxtacortical spots in patients with migraine. Eur J Neurol 2012;19:1086-92.

57. Park HK, Lee SY, Kim SE, Yun CH, Kim SH. Small deep white matter lesions are associated with right-to-left shunts in migraineurs. J Neurol 2011;258:427-33.

58. Iwasaki A, Suzuki K, Takekawa H, Takashima R, Suzuki A, Suzuki S, et al. The relationship between right-to-left shunt and brain white matter lesions in Japanese patients with migraine: a single center study. J Headache Pain 2017;18:3.

59. Debette S, Markus HS. The clinical importance of white matter hyperintensities on brain magnetic resonance imaging: systematic review and meta-analysis. BMJ 2010; 341:c3666.

60. Wen K, Nguyen NT, Hofman A, Ikram MA, Franco OH. Migraine is associated with better cognition in the middle-aged and elderly: the Rotterdam Study. Eur J Neurol 2016;23:1510-6.

61. Baars MA, van Boxtel MP, Jolles J. Migraine does not affect cognitive decline: results from the Maastricht aging study. Headache 2010;50:176-84.

62. Rist PM, Kang JH, Buring JE, Glymour MM, Grodstein F, Kurth T. Migraine and cognitive decline among women: prospective cohort study. BMJ 2012;345:e5027.

63. Xie H, Zhang Q, Huo K, Liu R, Jian ZJ, Bian YT, et al. Association of white matter hyperintensities with migraine features and prognosis. BMC Neurol 2018;18:93.

64. Park BY, Lee MJ, Lee SH, Cha J, Chung CS, Kim ST, et al. DEWS (DEep White matter hyperintensity Segmentation framework): a fully automated pipeline for detecting small deep white matter hyperintensities in migraineurs. Neuroimage Clin 2018;18:638-47.

65. Hong J, Park BY, Lee MJ, Chung CS, Cha J, Park H. Twostep deep neural network for segmentation of deep white matter hyperintensities in migraineurs. Comput Methods Programs Biomed 2020;183:105065.

66. Sam K, Peltenburg B, Conklin J, Sobczyk O, Poublanc J, Crawley AP, et al. Cerebrovascular reactivity and white matter integrity. Neurology 2016;87:2333-9.

67. Tuladhar AM, van Norden AG, de Laat KF, Zwiers MP, van Dijk EJ, Norris DG, et al. White matter integrity in small vessel disease is related to cognition. Neuroimage Clin 
2015;7:518-24.

68. Fazekas F, Ropele S, Enzinger C, Gorani F, Seewann A, Petrovic K, et al. MTI of white matter hyperintensities. Brain 2005; 128:2926-32.

69. Arkink EB, Palm-Meinders IH, Koppen H, Milles J, van Lew $\mathrm{B}$, Launer LJ, et al. Microstructural white matter changes preceding white matter hyperintensities in migraine. Neurology 2019;93:e688-94.

70. Heye AK, Culling RD, Valdes Hernandez Mdel C, Thrippleton MJ, Wardlaw JM. Assessment of blood-brain barrier disruption using dynamic contrast-enhanced MRI: a systematic review. Neuroimage Clin 2014;6:262-74.
71. Hougaard A, Amin FM, Christensen CE, Younis S, Wolfram $\mathrm{F}$, Cramer SP, et al. Increased brainstem perfusion, but no blood-brain barrier disruption, during attacks of migraine with aura. Brain 2017;140:1633-42.

72. Amin FM, Hougaard A, Cramer SP, Christensen CE, Wolfram F, Larsson HBW, et al. Intact blood-brain barrier during spontaneous attacks of migraine without aura: a 3T DCE-MRI study. Eur J Neurol 2017;24:1116-24.

73. Kim YS, Kim M, Choi SH, You SH, Yoo RE, Kang KM, et al. Altered vascular permeability in migraine-associated brain regions: evaluation with dynamic contrast-enhanced MRI. Radiology 2019;292:713-20. 